\title{
Performance Research of a New Generation of Intelligent Substation Communication Network
}

\author{
Lifeng Liu ${ }^{1, \text { a) }}$, Ning Wang ${ }^{2, \text { b) }}$, Yong $\mathrm{Li}^{1, \mathrm{c})}$, Ping $\mathrm{Yu}^{2, \mathrm{~d})}$ \\ ${ }^{1}$ State Grid Shaoxing Power Company, Shaoxing 312000, China; \\ ${ }^{2}$ School of Electric and Electronic Engineering, North China Electric Power University, Baoding 071003, China \\ a)17260587@qq.com \\ b) Corresponding author: wangningncepu@163.com \\ c)52824228@qq.com \\ d) yuping63119@ncepu.edu.cn
}

\begin{abstract}
In view of the problems of network time synchronization, transmission delay and large jitter in the smart substation communication system, the multiplexing mode of communication network in smart substation is studied and the WDM network architecture mode is proposed. A new generation of smart substation WDM passive optical communication network architecture based on passive optical network and virtual point-to-point bidirectional transmission links, the use of business isolation technology to ensure the stability and reliability of the entire network. By simulation, the performance indexes of WDM passive optical network are obtained and analyzed. It is proved that the requirements of substation business to communication network can be realized through WDM communication network. Fully proved that the communication network can be applied to the substation communication network.
\end{abstract}

Key words: WDM; synchronization; communication system; network. point-to-point.

\section{INTRODUCTION}

Communication network is an important part of smart substation. It undertakes the exchange of key information such as real-time data of power grid, acquisition, transmission, real-time control and clock synchronization of smart substations to realize important real-time control such as relay protection. The structure type of smart substation communication network is of great significance to ensure the safe operation of power grid [1]. At present, smart substation communication platform has two kinds of structures. The first is a smart substation [2] based on the industrial Ethernet switch technology, which uses multiple switch cascades to accommodate the interconnection and access of many devices of different functions. This kind of communication network structure relies on more resource redundancy to meet the requirements of queuing and time delay. The handling of broadcast storms and other issues is relatively simple and extensive. In some cases, the reliability of data transmission deteriorates and even serious packet loss phenomenon [3]. The second is a new generation of smart substation [4-5] communication network based on time-division multiplexing passive optical network architecture. This kind of network structure can save many cascaded switches, the network connection is greatly simplified, only one central switch is needed, and the use of packet transport network switching technology to complete the main exchange, to solve the congestion problem works well. The network structure is based on PON technology and needs accurate timing. Each device can only work in the specified timeslots and wait for a long time. To meet the requirements of real-time business, priority control is added to ensure the priority transmission such as the sampled value(SV) message.

Smart substation business requires high reliability and high real-time communication network. Literature [6-10] introduced the planning of a new generation of smart grids and put forward higher requirements on the performance of the network. The business messages need to be mutually independent, that is, business isolation [11]. In the data 
transmission, generic object-oriented substation event (GOOSE) messages, sampled value messages, manufacturing message specification (MMS) messages, address resolution protocol (ARP) messages and time messages are transmitted in the same communication network, an increase of network load. The transmission delay of the switching equipment is not fixed, and the network data is more complicated, which may bring new problems. In smart substation communication network, the sampling data synchronization need to rely on external time synchronization, and sampling data without synchronization may affect the safe operation of the substation [12-13].

\section{WAVELENGTH DIVISION MULTIPLEXING COMMUNICATION NETWORK ARCHITECTURE}

WDM technology and passive optical network technology combine to build a new type of smart substation communication network, which is based on the virtual point-to-point bidirectional transmission link network, with the advantages of the two technologies. The new substation communication network has obvious advantages in reducing transmission delay and improving network performance and reliability. The communication network architecture is shown in figure 1. A novel substation communication network connects an intelligent electronic device (IED) to a passive optical network through an optical network unit (ONU), and the ONU is connected to the central switch through the optical distribution network (ODN).

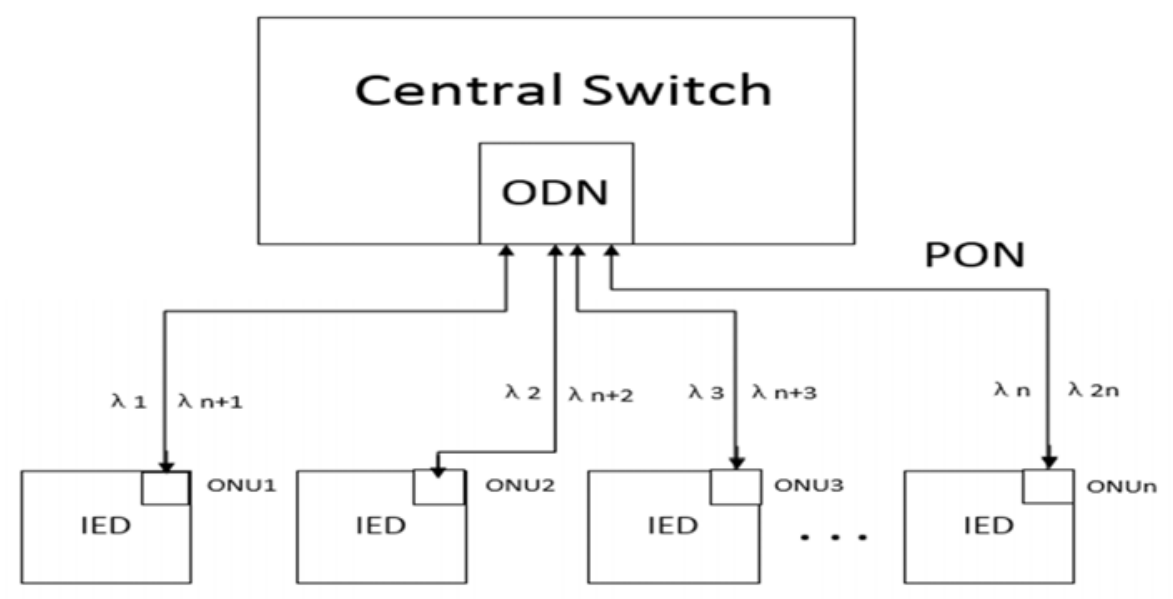

FIGURE 1. Based on WDM-PON smart substation communication network architecture

Each intelligent electronic device is connected to the ONU. Each ONU allocates a pair of wavelengths for uplink and downlink respectively. In this way, a bidirectional connection of the central switch to each ONU is established, and each intelligent electronic device is connected into the communication network. In the uplink transmission, the IED uploads the data to the ONU. Each ONU uses a specific wavelength to transmit business messages. Therefore, no timing and network synchronization are needed. During downlink transmission, the central switch adds data to carriers with different wavelengths through modulation and transmits them to ONU through wavelength routes respectively. This completes the bidirectional communication between the central switch and each device.

\section{NETWORKING TECHNOLOGY AND FEATURES}

The IEC 61850 standard [14] defines three kinds of business messages in the smart substation communication network: (1) Generic object-oriented substation event messages, including messages such as tripping, closing, blocking and unlocking. The switch in the substation changes, its status, position signal and blocking signal will be transmitted to the corresponding device through the GOOSE message, or the trip command issued by the protection device will also be sent to the corresponding intelligent terminal by the tripping GOOSE message. A sudden increase in the amount of information during system failure. (2) The sampled value message is generated by the merging unit, mainly deals with all kinds of sampled value data, and sends the various parameters to the equipment for protection, measurement and control. The total amount of messages is basically stable. (3) The manufacturing message 
specification message is mainly used for transmitting messages between substation level and bay level and between substation level devices. It has a large amount of data and is insensitive to delay but has strict requirements on data integrity.

After the business messages generated by different electronic devices enter the PON through the ONU, different business messages are carried by different wavelengths and do not interfere with each other during transmission. When the business messages are transmitted through the ODN, different wavelengths are multiplexed to a optical fiber, until the business data into the center switch. Substation business can realize substation unified network through different wavelengths in the same network, and realize full business isolation of MMS, SV and GOOSE. In this kind of network system, sending and receiving of each business message use different wavelengths, so it does not need network synchronization and improves the reliability of the communication system.

Network communication system uses multi-protocol label switching (MPLS) technology, MPLS is the use of tags for data forwarding. In the PON uplink transmission process, ONU must be strictly in accordance with the switch scheduling data transmission, at any time can be regarded as any one of the ONU and switch point-to-point transmission, will not conflict. But it requires a packet label indicating the label switching path (LSP) which the packet belongs. In downlink transmission, the data is broadcast, all ONU receive the same information. This time ONU need to identify the information, and ONU only receive their own data. According to the above reasons, the label domain and the ONU identification domain can be merged with the meaning of the label in the uplink and the ONU identification in the downlink [15].

\section{SIMULATION EXPERIMENT RESEARCH}

\section{Experimental Network Construction}

Experimental network completed by Opti system simulation, multi-mode fiber constitutes PON and connect the various terminal devices, the experimental network diagram in figure 2.

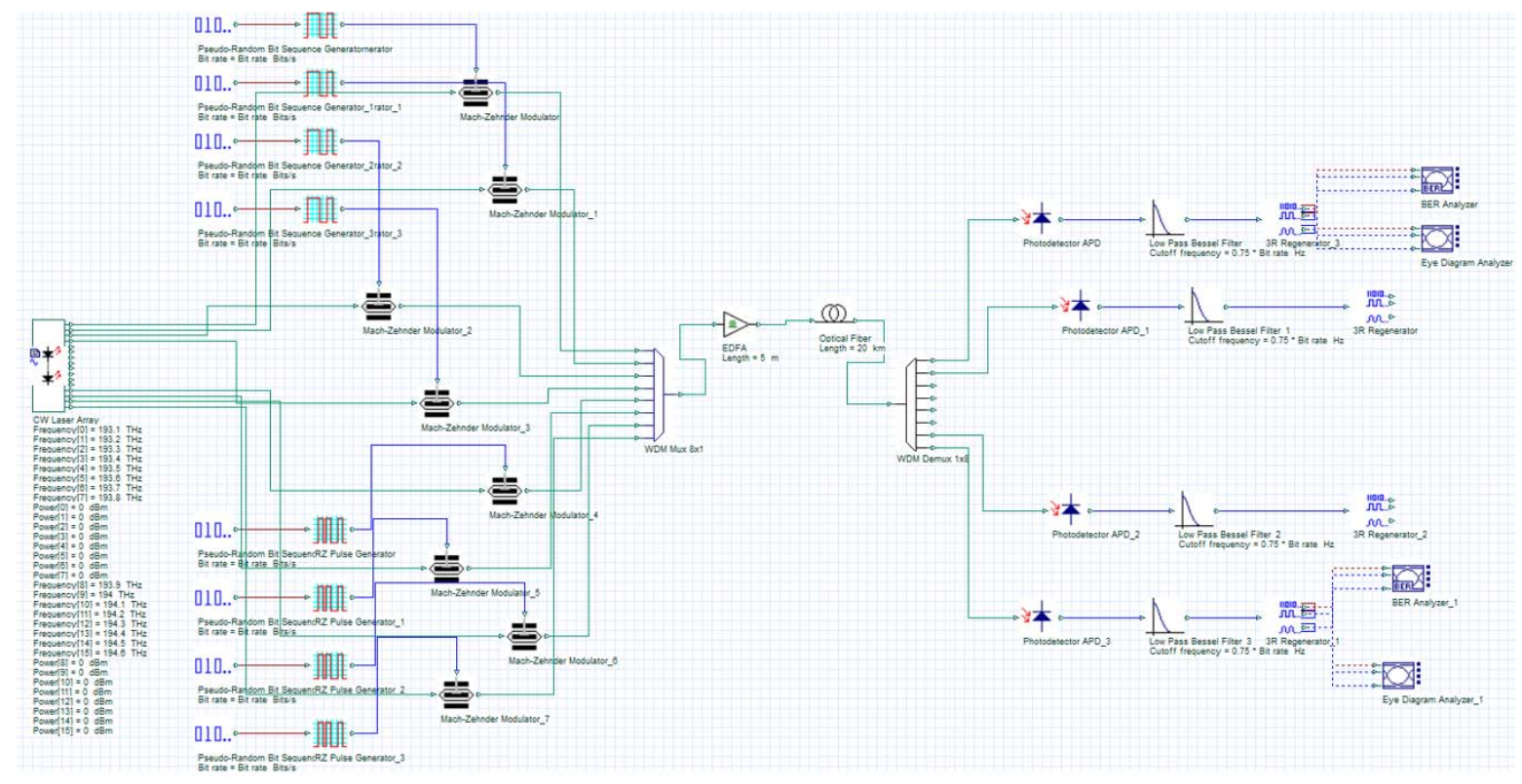

FIGURE 2. Experimental network diagram

As shown in figure 2, the experimental network uses a Mach-Zehnder modulator to modulate the CW Laser light, resulting in a total of eight carriers of different frequency wavelengths. The system generates a digital baseband signal through a random binary code generator and a not return to zero pulse generator that modulates the optical carrier generated by the laser through a modulator to produce eight light sources. In the multiplexer, eight optical signals with a frequency of $0.1 \mathrm{THZ}$ are combined into a optical fiber. After $20 \mathrm{~km}$ of optical fiber transmission, the transmission 
channel also includes EDFA, optical fiber and other components. At the receiving end, demultiplexer restore the original signal.

\section{Main Performance Indicators}

\section{Throughput}

Throughput refers to the network, equipment, ports, virtual circuits or other facilities, the number of successfully transmitted data per unit of time. The throughput here refers to the link bandwidth between devices in the communication network system. In order to ensure the quality of service and to achieve zero congestion in the communication network, the ONU upstream bandwidth is set to a fixed bandwidth of $128 \mathrm{M}$ and the peak bandwidth to $200 \mathrm{M}$, through the rational design and planning and using the TDM-PON technology.

\section{Bit Error Rate Performance}

Bit error rate(BER) is a measure of communication system performance, one of the important indicators. The analysis of the bit error rate existing in the WDM-PON mainly involves the problem of the interference between different wavelengths in the optical multiplexer in the system and can be studied by means of eye diagram and Q factor. In the simulation result of WDM system, the eye diagram is one of the important means to test the reliability of the system, and the system transmission performance can be directly observed. The Q factor is an important indicator of signal quality and system performance in wavelength division multiplexed systems. Higher $Q$ factor value mean better BER.

As shown in figure 3, the eye diagram with the frequency of $193.1 \mathrm{THZ}$ can be found to be thick due to noise and jitter, but the entire eye diagram is still clear with high noise margin and good anti-interference performance.

圆

Eye Diagram Analyzer

Dы Click On Objects to open properties. Move Objects with Mouse Drag

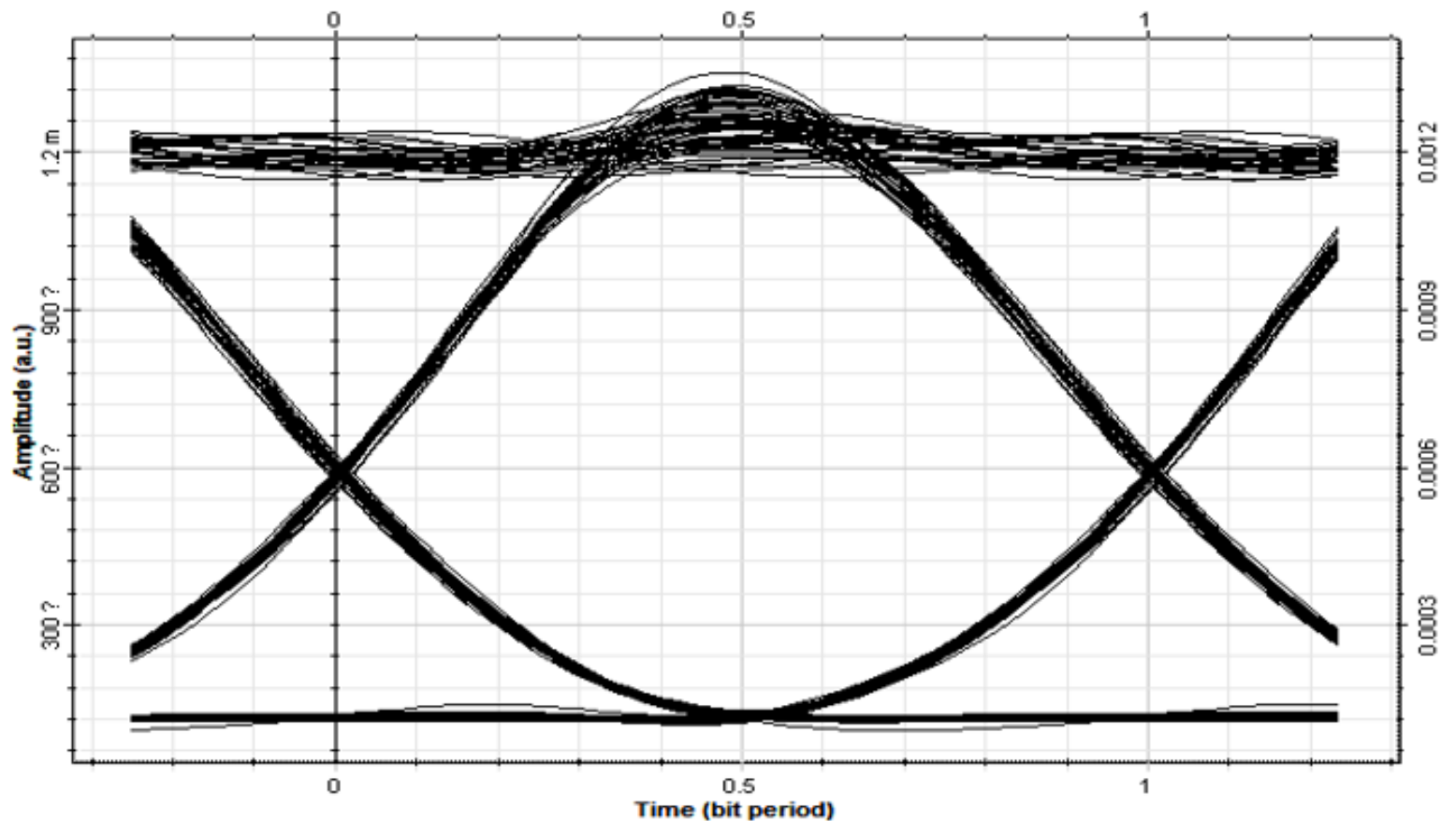

FIGURE 3. Eye Diagram 


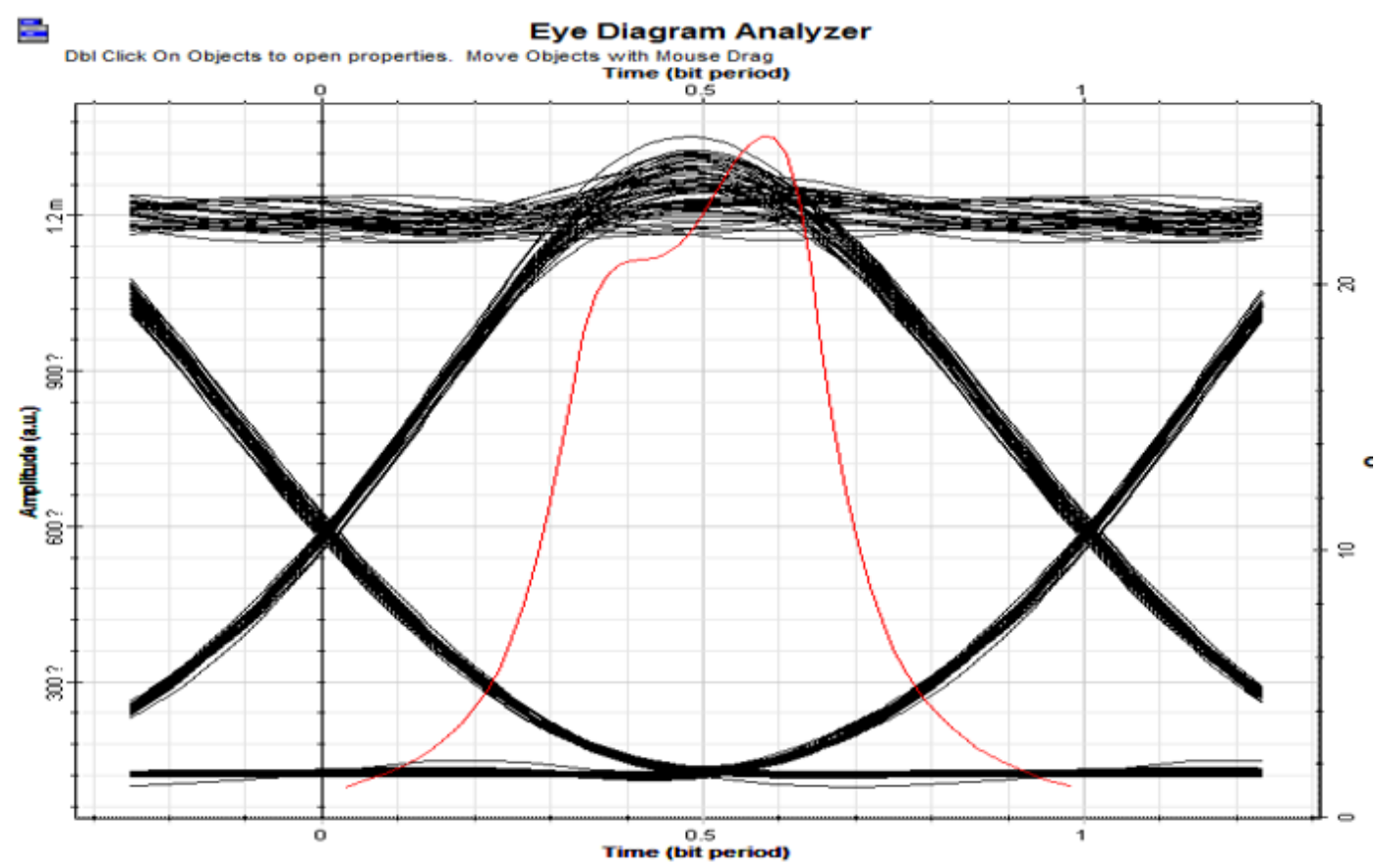

FIGURE 4. Q factor and eye diagram

The relationship between $\mathrm{Q}$ factor and eye diagram is shown in figure 4 . The red line in figure 4 represents the $\mathrm{Q}$ factor. As can be seen from figure 4, the larger Q factor value in the eye opening. Normally, the BER is about $10^{-9}$ when $\mathrm{Q}=6$ and about $10^{-12}$ when $\mathrm{Q}=7$. The communication network has a larger judgment range under the condition of ensuring system error rate requirement $\left(<10^{-12}\right)$. As can be seen from figure 4 , at the best sampling time of the eye diagram, the $\mathrm{Q}$ factor value can already approach 23 . This shows that the signal-to-noise ratio is high currently, and there is almost no distortion and crosstalk in the transmitted signal.

\section{Delay Performance}

Send business messages through the OPNET simulated ONU and test the delay of different messages. After several tests and each teste no more than 24 hours, recording the maximum delay, the minimum delay and the average delay of each business respectively.

TABLE 1. Different business message delay and message loss rate comparison

\begin{tabular}{|c|c|c|c|}
\hline Message Type & 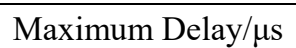 & Minimum Delay $/ \mu \mathrm{s}$ & Average Delay/ $\mu \mathrm{s}$ \\
\hline GOOSE & 689.76 & 23.65 & 264.25 \\
\hline SV & 743.21 & 62.24 & 317.02 \\
\hline MMS & 1402.38 & 472.62 & 880.67 \\
\hline
\end{tabular}

Table 1 compares the delay of three business messages. As can be seen from the table, when the network transmits messages, the network delay is far less than the specified value (the GOOSE message transmission delay is 3 to 10 MS, the total transmission delay of SV messages is less than $50 \mathrm{MS}$, and the MMS messages generally do not exceed $500 \mathrm{MS})$

\section{CONCLUSION}

Through the simulation of WDM-PON and virtual point-to-point transmission link, the ideal eye diagram and Qfactor curve are obtained, which verifies the new WDM passive optical communication network for the smart 
substation can achieve business isolation and greatly guarantee the reliability of the network. The above results simplify the communication network structure of a smart substation, reduce construction costs, improve network reliability and real-time performance, and provide strong support for the construction of a new generation of smart substation communication networks.

\section{REFERENCES}

1. Zhu Lin, Wang Pengyuan, Shi Dongyuan. Description of state monitoring information model and its configuration for communication network in smart substations [J]. Automation of Electric Power Systems, 2013, 37 (11): 87-92.

2. Liu Pin, Chen Li. Application of Industrial Ethernet Technology in Substation Automation System [J]. Power System Protection and Control, 2008, 36 (23): 65-68 (in Chinese).

3. Wang Haizhu, Cai Zexiang, Zhang Yanxu, et al. Qualification exchange technology for improving real-time and reliability of information flow in intelligent substation [J]. Electric Power Automation Equipment, 2014, 34 (5): 156-162.

4. Ling Guang, Xu Weiguo, Wang Zhiliang, et al. Application of EPON + -based fixed-delay network with high reliability in smart substation [J]. Power System Protection and Control, 2016 (14): 89-94 (in Chinese).

5. Sun Haibo, Jin Naizheng, Ma Ping, et al. Key technologies of a new generation of intelligent substation communication network [J]. Optical Communication Research, 2017 (4): 1-3.

6. Liu Zhenya. China Electric Power and Energy [M]. 1 version. Beijing: China Electric Power Press, 2012: 1-42.

7. UHV "Veins" - Liu Zhenya, general manager of UHV power grid excerpt [J]. National Grid, 2006, 09: 23-25.

8. Technical Requirements for Expanding Demonstration Engineering of a New Generation of Smart Substation in 2014 (State Grid Smart [2014] No. 867).

9. "2014 new generation of intelligent substation expansion demonstration project construction plan"

10. "State Grid Corporation" Twelfth Five-Year "smart grid planning"

11. Wang Qiang, Xu Xiaolan, Zhang Jian. Research on new security isolation technology for intelligent substation communication service [J]. Power System Protection and Control, 2015,43 (17): 139-144 (in Chinese).

12. Zhang Yancang. New technology of sampling synchronization in intelligent substation network [J]. Electric Power Automation Equipment, 2015, 35 (8): 143-147.

13. Yang Gui, Lu Hang, Yuan Zhibin, et al. Research and implementation of flow control and synchronization method for process layer network in smart substation [J]. Power System Protection and Control, 2015 (11): 70 74.

14. International Standard IEC61850-2004, Communication networks and systems in substation[S].

15. Guo Yong. Research on Intelligent Passive Optical Network Based on GMPLS [D]. Shanghai Jiao Tong University, 2006. 